\title{
Synthesis of Subband Hybrid Filter Banks ADCs with Finite Word-length Coefficients using Adaptive Equalization
}

\author{
Zhiguo Song, Caroline Lelandais-Perrault (Member, IEEE), \\ Daniel Poulton and Philippe Bénabes (Member, IEEE) \\ Department of Signal Processing and Electronic Systems \\ SUPELEC \\ 91192 Gif sur Yvette, France \\ Email: firstname.lastname@supelec.fr
}

\begin{abstract}
Subband Hybrid Filter Banks (SHFB) ADCs are able to convert one or several narrow subbands among a given wideband signal. That provides an appropriate solution to "flexible spectrum" management for cognitive radio applications. In this paper, a SHFB ADC delivering a complex signal is considered. The optimal values of the digital coefficients are obtained thanks to an adaptive equalization method. The impact of digital coefficients quantization to the SHFB is studied. For a reconstruction quality target, the minimum quantization step is determined. This is validated in time and frequency domain with simulation results.
\end{abstract}

\section{INTRODUCTION}

Future cognitive radio systems should be able to adapt themselves to a multi-standard environment [1]. To achieve that, high performances ADCs are necessary. They should convert alternately a wideband signal with a low resolution for "sensing" purpose, and any narrow band signal with a higher resolution in a communication mode [2]. Hybrid Filter Banks (HFB) ADC seems to be a suitable solution for these requirements. Such a parallel architecture consists of an analog frequency decomposition (analysis bank), a conversionstage (ADC bank) and a digital reconstruction (synthesis bank). From a given ADC sampling rate, an HFB ADC can enlarge the conversion bandwidth and perform bandpass sampling. Also, HFB can convert a narrow bandwidth with a higher resolution by reconfiguring its digital part. This can be achieved with a Subband HFB (SHFB) proposed in [3]. However, SHFBs are very sensitive to analog errors that mainly occur while manufacturing [3]. To solve this problem, an adaptive equalization method proposed in [4] finds the digital coefficients corresponding to the analog filter bank. Figure 1 shows the principle of the adaptive equalization method to synthesize a SHFB. A well chosen test signal is given at the input of the SHFB. The output of the SHFB is compared to the desired signal linked to the test signal. The error is used to correct the digital filter coefficients iteratively thanks to the Least Mean Square (LMS) algorithm [5]. When the error decreases so much that the reconstruction accuracy is achieved, the synthesis process is stopped. Practically, in an embedded system, the test signal and the desired signal samples may be stored in a memory.

In this paper, the SHFB ADC digital coefficients are Finite Word-Length (FWL) in complex form. The quantization

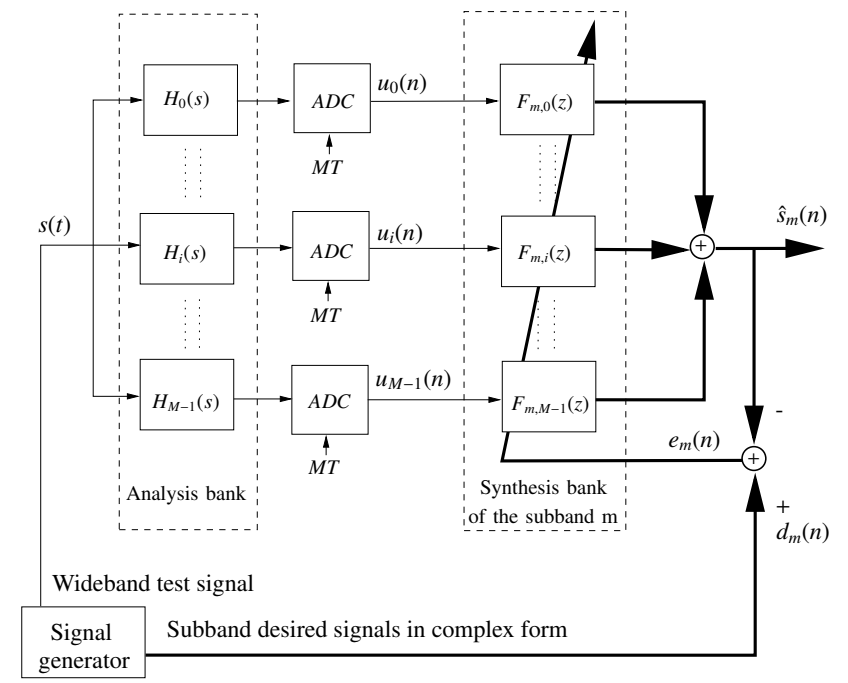

Fig. 1. Adaptive equalization for synthesizing of $M$-channel SHFB ADC, the subscripts $m$ and $i$ designate respectively the subband number and the channel number. $m, i \in[0, M-1]$. $T$ denotes the Nyquist sampling period of the input signal. The thick line designates the signal in complex form.

influences upon the subband reconstruction and the adaptive equalization are studied. By considering the test signal characteristics and a bank that contains ideal analog filters, a theoretical number of bits coding the digital coefficients may be determined function to the effective resolution of the SHFB.

In section II, the generations of the test signal $s(t)$ and the subband desired signal $d_{m}(n)$ in complex form are presented. Then, the synthesis method using adaptive equalization is justified theoretically for the SHFB ADC. In section III, the quantization influences of the digital coefficients are studied. The theoretical minimum number of quantization bits, which allows the SHFB achieve the required effective resolution is pointed out. In section IV, initializing the digital FWL coefficients to zero, an eight-channel SHFB ADC is simulated in both time and frequency domains. Based on the simulation results, the performances of the SHFB ADC are discussed.

\section{Adaptive equalization for SYNTHESIZING a SHFB ADC}

The main originality of the adaptive equalization method applied to the SHFB synthesis relies upon the choice of the 
test and desired signals. The adaptive algorithm is the classical complex LMS algorithm [5].

\section{A. Test signal $s(t)$ and desired signal $d_{m}(n)$}

Let $B W$ and $T$ denote respectively the bandwidth and the Nyquist sampling period of $s(t) .2 \pi \Omega_{m}$ designates the central frequency of the subband $m$, which bandwidth $B W_{s u b}=\frac{2 B W}{M}$. Let $G(j \Omega)$ designate the frequency response of a lowpass filter (LF), which bandwidth is equal to $B W_{s u b}$. In order to avoid the additional interferences generated by the frequencies apart the passband, the LF's selectivity should be high [6]. After being modulated by $e^{j \Omega_{m}}$, the LF is transfered to complex bandpass filters (CBF). As shown in Figure 2, a pseudo zero-mean white noise signal filtered by the CBFs, the subband signal $S_{m}(j \Omega)$ is obtained. Then, the test signal is achieved by $\sum_{m=0}^{M-1} S_{m}(j \Omega)$, which variance is noted by $\sigma_{s}^{2}$. Let denote $\sigma_{s_{m}}^{2}$ the variance of subband signal, since $s(t)$ consists $M$ subband signals, we have

$$
\sigma_{s_{m}}^{2}=\frac{\sigma_{s}^{2}}{M}
$$

Thanks to the complex bandpass sampling, $S_{m}(j \Omega)$ is downconverted directly to obtain the desired signal $d_{m}(n)$ with aliasing free.

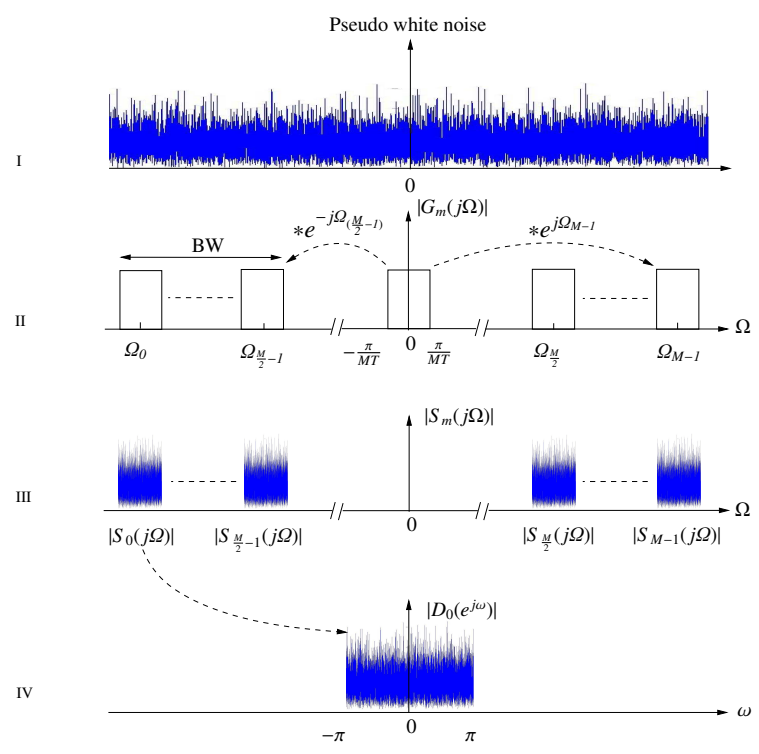

Fig. 2. Generations of the test signal $s(t)$ and $d_{m}(n)$. The spectra of : (I) a pseudo white noise, (II) transferring the lowpass filter to the bandpass filters, (III) the test signal $s(t)$ and (IV) the desired signal of the subband $0: d_{0}(n)$.

\section{B. Adaptive synthesis of the complex SHFB ADC}

The digital reconstruction of the subband $m$ through the SHFB ADC can be presented in frequency-domain by

$$
\hat{S}_{m}\left(e^{j \omega}\right)=\sum_{p=-(M-1)}^{M-1} S\left(j\left(\Omega-\frac{2 \pi p}{M T}\right)\right) T_{m, p}\left(e^{j \omega}\right)
$$

with the reconstruction function $T_{m, p}$ that is defined by

$$
T_{m, p}\left(e^{j \omega}\right)=\sum_{i=0}^{M-1} H_{i}\left(j\left(\Omega-\frac{2 \pi p}{M T}\right)\right) F_{m, i}\left(e^{j \omega}\right)
$$

where $H_{i}$ denotes the transfer function of analysis filter in the channel $i, F_{m, i}$ designates the transfer function of the synthesis filter in subband $m$ channel $i$. $\Omega$ means the pulsation and its normalization defined by $\omega=\Omega T$. For the purpose of the SHFB's perfect reconstruction, the sum of the aliasing terms $T_{m, p \neq 0}$ should be 0 , and the distortion term $T_{m, p=0}$ must satisfy [3]

$$
T_{m, 0}\left(e^{j \omega}\right)=\sum_{i=0}^{M-1} H_{i}\left(j \frac{\omega}{T}\right) F_{m, i}\left(e^{j \omega}\right)=e^{-j \omega \rho} \quad \rho \in \mathbb{R}_{*}^{+}
$$

Let $\mathbf{u}(n)$ designate all the inputs of the synthesis bank, we have

$$
\mathbf{u}(n)=\left[\mathbf{u}_{0}(n), \cdots, \mathbf{u}_{i}(n), \cdots, \mathbf{u}_{M-1}(n)\right]^{T},
$$

where $\mathbf{u}_{i}(n)$ denotes the inputs of the channel $i, \mathbf{v}^{T}$ means the transpose of the vector $\mathbf{v}$. $L$-tap finite impulse response (FIR) filters are used in the synthesis bank, so, $\mathbf{u}_{i}(n)=\left[u_{i}(n), \cdots, u_{i}(n-l), \cdots, u_{i}(n-L+1)\right], l \in[0, L-1]$.

The complex synthesis filters coefficients in the the subband $m$ channel $i$ may denoted by the vector $\tilde{\mathbf{f}}_{m, i}=\left[\tilde{f}_{m, i}(0), \cdots, \tilde{f}_{m, i}(l), \cdots, \tilde{f}_{m, i}(L-1)\right]$. During the synthesis process, the coefficient $\tilde{f}_{m, i}(l)$ is adjusted to minimize the reconstruction error iteratively. Therefore, the vector that contains all of the synthesis coefficients of the subband $m$ may be given by

$$
\tilde{\mathbf{f}}_{m}=\left[\tilde{\mathbf{f}}_{m, 0}, \cdots, \tilde{\mathbf{f}}_{m, i}, \cdots, \tilde{\mathbf{f}}_{m, M-1}\right]
$$

Hence, the SHFB ADC's estimated output $\hat{s}_{m}(n)$ and the reconstruction error $e_{m}(n)$ can be represented as follows

$$
\begin{aligned}
\hat{s}_{m}(n) & =\tilde{\mathbf{f}}_{m}^{*} \mathbf{u}(n) \\
e_{m}(n) & =d_{m}(n)-\hat{s}_{m}(n)
\end{aligned}
$$

where "*" denotes the operator of conjugation. We define $\mathbf{J}=\mathbb{E}\left[\left|e_{m}(n)\right|^{2}\right]$ the cost function for the reconstruction of subband $m$, where $\mathbb{E}$ means the operator of expectation. According to [4], the optimal synthesis coefficients can be approximated by applying the complex LMS algorithm [5]. The iterative adjustments of the coefficients in complex form are given as follows

$$
\begin{array}{r}
\tilde{\mathbf{f}}_{m}^{I}(n+1)=\tilde{\mathbf{f}}_{m}^{I}(n)+\mu e_{m}^{I}(n) \mathbf{u}(n) \\
\tilde{\mathbf{f}}_{m}^{Q}(n+1)=\tilde{\mathbf{f}}_{m}^{Q}(n)+\mu e_{m}^{Q}(n) \mathbf{u}(n)
\end{array}
$$

where $\mu$ is named step-size, " $\mu e_{m}(n) \mathbf{u}(n)$ " are considered as correction term. The superscripts $I$ and $Q$ designate "in-phase" and "quadrature" components, that is real and imaginary parts of the complex number respectively. The implementation of (9) and (10) in the synthesis bank is presented in Figure 3. The condition of stability of the implemented complex LMS [5] in the SHFB ADC context can be defined by [4] 


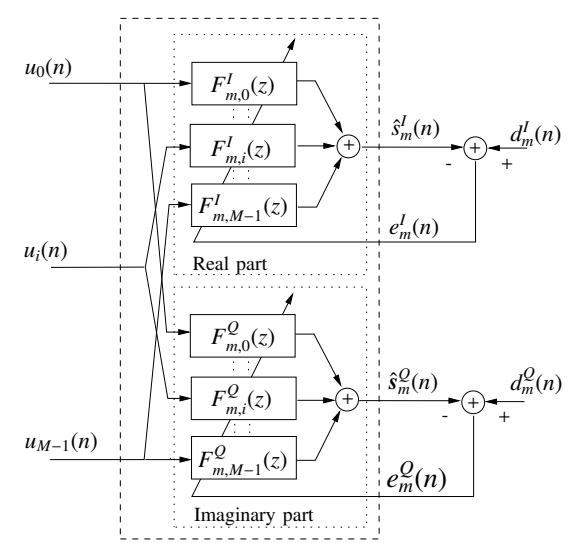

Fig. 3. Synthesis of the complex SHFB ADC by using the complex LMS algorithm.

$$
0<\mu<\frac{1}{M L \mathbb{E}\left[u_{i}^{2}(n)\right]}=\frac{1}{M L \sigma_{u}^{2}}
$$

where $\mathbb{E}\left[u_{i}^{2}(n)\right]$ denotes the expectation of the power spectral density of the synthesis bank inputs. Since the test signal is supposed to be zero-mean, and so does $\mathbf{u}(n)$. Thus, we have $\sigma_{u}^{2}=\mathbb{E}\left[u_{i}^{2}(n)\right]$. Futhermore, $s(t)$ is decomposed into $M$ channels by the analog filters which are assumed without overlap, the relationship between $\sigma_{u}^{2}$ and $\sigma_{s}^{2}$ can be represented by

$$
\sigma_{u}^{2}=\frac{\sigma_{s}^{2}}{M}
$$

Let substitute (12) in (11), we have

$$
\mu_{\max }<\frac{1}{L \sigma_{s}^{2}}
$$

III. INFLUENCES OF SYNTHESIS COEFFICIENT QUANTIZATION

\section{A. Degradation of the $S H F B$ ADC's reconstruction accuracy}

Let define the following model to represent the complex synthesis coefficients in the subband $m$

$$
\tilde{\mathbf{f}}_{m}=\mathbb{Q}\left[\tilde{\mathbf{f}}_{m}\right]+\tilde{\boldsymbol{\epsilon}}
$$

where the vectors $\mathbb{Q}\left[\tilde{\mathbf{f}}_{m}\right]$ and $\tilde{\epsilon}$ denote respectively the FWL FIR coefficients and quantization errors, $\mathbb{Q}[]$ means quantization operator. Let introduce (14) into (7), then substitute (7) into (8), we have

$$
e_{m}(n)=d_{m}(n)-\mathbb{Q}\left[\tilde{\mathbf{f}}_{m}^{*}\right] \mathbf{u}(n)-\tilde{\epsilon} \mathbf{u}(n)
$$

Hence, the degradation of the reconstruction accuracy due to quantization errors can be presented by

$$
\Delta_{e_{m}(n)}=\tilde{\epsilon} \mathbf{u}(n)=\sum_{i=0}^{M-1} \sum_{l=0}^{L-1} \tilde{\epsilon}_{i}(l) u_{i}(n-l)
$$

where $\tilde{\epsilon}_{i}$ and $u_{i}$ are assumed to be independent random zeromean values, so, the variance of $\Delta_{e_{m}(n)}$ can be given by

$$
\sigma_{\Delta_{e_{m}}}^{2}=M L \sigma_{\tilde{\epsilon}}^{2} \sigma_{u}^{2}
$$

In order to simplify the demonstration thereafter, the analysis and synthesis filters are supposed to be ideal bandpass filters. Assuming that the gain of $H_{i}(s)$ is equal to 1 [7], the gain of $F_{m, i}(z)$ should be 1 according to (4). Let $B_{m}$ denote the number of bits coding the digital coefficients. Since the maximum absolute value of the synthesis coefficients is 1 , the quantization errors can be given by

$$
\sigma_{\tilde{\epsilon}}^{2}=\frac{\left(\frac{1}{2^{B_{m}-1}}\right)^{2}}{12}=\frac{1}{3 \times 2^{2 B_{m}}}
$$

Let introduce (12) and (18) into (17), and according to (1), we have

$$
\sigma_{\Delta_{e_{m}}}^{2}=\frac{L \sigma_{s}^{2}}{3 \times 2^{2 B_{m}}}=\frac{M L \sigma_{s_{m}}^{2}}{3 \times 2^{2 B_{m}}}
$$

Usually, the quantization noise is measured in term of signal-to-noise ratio (SNR) which is defined as following for the subband $m$

$$
S N R_{m}=10 \log _{10}\left[\frac{\sigma_{s_{m}}^{2}}{\sigma_{e_{m}}^{2}}\right] \approx 6 N_{m}
$$

where $N_{m}$ designates the effective resolution of the subband $m$ in bit. For the reconstruction of SHFB ADC with an expected resolution $N_{m}$, the following relationship must be satisfied

$$
\sigma_{e_{m}}^{2} \approx \frac{\sigma_{s_{m}}^{2}}{10^{0.6 N_{m}}} \gg \sigma_{\Delta_{e_{m}}}^{2}
$$

According to (19) and (21), the estimated quantization number of bits $\hat{B}_{m}$ coding the synthesis coefficients should be

$$
\hat{B}_{m} \gg 0.5 \log _{2}\left[\frac{M L 10^{0.6 N_{m}}}{3}\right]
$$

\section{B. Effect to the adaptive equalization}

Due to quantization errors, the adjustments of the coefficients can continue in the condition of the LMS correction term is bigger than half of the quantization interval [8]. This may be presented by

$$
\left|\mu e_{m}(n) u_{m}(n-l)\right|>\frac{1}{2^{B_{m}}}
$$

The condition above can be approximated by the root mean square value of their magnitudes. That is

$$
\mu \sigma_{e_{m}} \sigma_{u}>\frac{1}{2^{B_{m}}}
$$

Let substitute $\sigma_{u}$ by (12) and $\mu$ by (13) into (24). Then, according to (1) and (20), we obtain the estimated minimum value of $\hat{B}_{m}$

$$
\hat{B}_{m}^{m i n}>3.33\left[\log _{10}(M L)+0.3 N_{m}\right]
$$

$\hat{B}_{m}^{\min }$ allows the LMS algorithm to continue the adjustments of $\mathbb{Q}\left[\tilde{\mathbf{f}}_{m}\right]$ to approach their optimal values $\mathbb{Q}\left[\mathbf{f}_{m}^{o p t}\right]$. Hence, the SHFB's reconstruction accuracy can be satisfied with $\mathbb{Q}\left[\mathbf{f}_{m}^{\text {opt }}\right]$. 


\section{Simulation Results}

\section{A. Conditions of simulation}

Considering the wideband signal to convert is in the frequency band $\left[\begin{array}{ll}0.85 & 1.25\end{array}\right] \mathrm{GHz}$. The analog signal is split into a bank of analog bandpass filters which consists in eight pure Gm-LC resonators [2]. Their central frequencies and quality factors are respectively $2 \pi \Omega_{i}$ and $Q_{i}$. The transfer function of the analysis filter of each channel is given by

$$
\mathbf{H}_{i}(s)=\frac{\frac{\Omega_{i}}{Q_{i}} s}{s^{2}+\frac{\Omega_{i}}{Q_{i}} s+\Omega_{i}^{2}}
$$

A pseudo random sequence is used as a source signal, the test signal and desired signal are generated by following the process described in the section II.A. In order to reduce the aliasing terms, it is efficient to allocate a small part of frequency borders (low and high frequencies) at the spectrum as guard band (GB) [4], which is equal to $7 \%$ of the bandwidth in this paper. The adaptive synthesis process is performed upon the subband 0 . The reconstruction accuracy is defined by $S N R_{0}$ that is expected to be $82 \mathrm{~dB}$, i.e. an effective resolution of $N_{0}=14$. That should satisfy the requirements of communication mode like GSM [9]. According to (22) and (25), the estimated $\hat{B}_{0}^{\text {min }}>24$ bit, then $\hat{B}_{0}^{\text {min }}$ is equal to $25 \mathrm{bit}$.

\section{B. Simulation results of synthesis in time domain}

The digital coefficients in complex form are initialized to zero. The synthesis process of the SHFB ADC is simulated in the time-domain with Matlab using $\mathrm{C}$ MEX-functions. With different $B_{0}$ values, several simulations are performed and the results are shown in Figure 4. To achieve the required resolution of 14 bit, the experimental minimum quantization number of bits, noted by $B_{0}^{\text {min }}$, is equal to 28 bit. $B_{0}^{\text {min }}$ exceeds here its theoretical value of 3 bit. Indeed, in the theoretical proof in section III, the analog filters have been assumed ideal with uniform frequency response in the appreciate subband. These hypotheses can be satisfied so hardly that they cause a degradation compared to the theoretical result. With $B_{0}=28$ bit, in the end of the $800^{\text {th }}$ iteration, the synthesis process meets the required resolution of 14 bit. And from then, the LMS cannot continue the adjustments of the coefficients. Thus, we can stop the process. The saved coefficients can be considered having their optimal quantized values noted by $\mathbb{Q}\left[\mathbf{f}_{0}^{\text {opt }}\right]$.

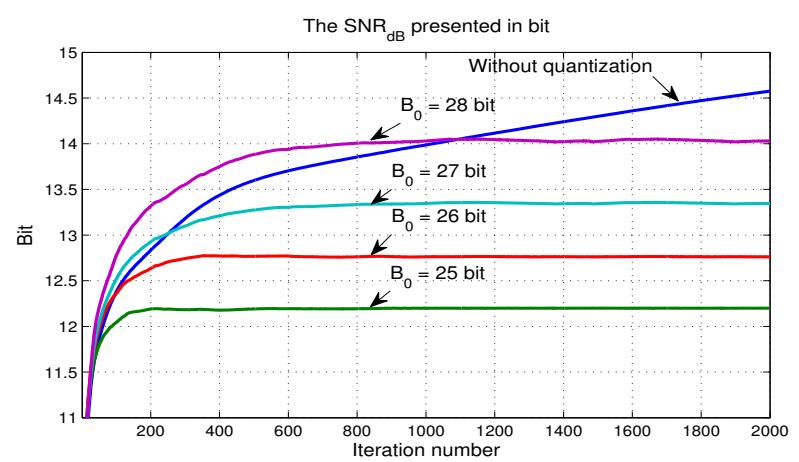

Fig. 4. Influence of digital coefficient quatization to the SHFB ADC's reconstruction accuracy and adaptive equalization.

\section{Validation in frequency domain}

Fixing $\mathbb{Q}\left[\mathbf{f}_{0}^{o p t}\right]$ and introducing their frequency responses $F\left(e^{j \omega}\right)$ into the reconstruction function (3), the distortion term and the sum of the aliasing terms can be calculated in decibel (Figure 5). Their mean values are $\mathbb{E}\left[\left(T_{0,0}\right)_{d B}\right]=1 \times 10^{-5} d B$ and $\mathbb{E}\left[\sum\left(T_{0, p \neq 0}\right)_{d B}\right]=-85 d B$ respectively. Hence, the performance of the SHFB can be presented by the Mean Distortion-toAliasing Ratio (MDAR) [4]. We have $M D A R_{d B}=\mathbb{E}\left[\left(T_{0,0}\right)_{d B}\right]-$ $\mathbb{E}\left[\sum\left(T_{0, p \neq 0}\right)_{d B}\right]=85 d B$, i.e. a resolution of 14.1 bit. This presents an accordance with the simulation results in time domain.

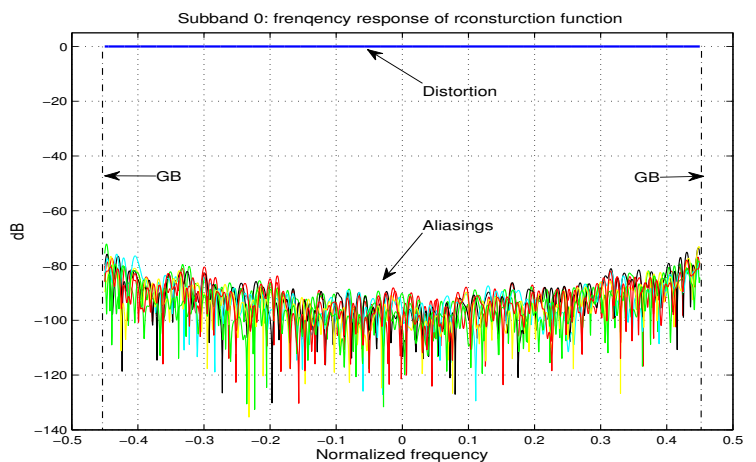

Fig. 5. Frequency response of the SHFB ADC with $\mathbb{Q}\left[\mathbf{f}_{0}^{\text {opt }}\right]$, where $B_{0}=28$ bit.

In this paper, the influence of digital coefficient quantization to the SHFB's reconstruction is studied. In order to guarantee the subband reconstruction accuracy by using the adaptive equalization, the minimum number of bits coding coefficient is theoretically pointed out. Due to the hypotheses are not be satisfied practically, it needs supplemental 3 bits over the theoretical value to obtain an effective resolution of 14 bit on the SHFB's output. Thanks to the simplicity of the LMS algorithm, the adaptive synthesis process makes the SHFB realistic for cognitive radio applications, such as "flexible spectrum" management.

\section{REFERENCES}

[1] B. Fette(Editor), "Cognitive radio technology" Newnes Elsevier, 2006.

[2] C. Lelandais-Perrault, T. Petrescu, D. Poulton, P. Duhamel, J. Oksman, "Wide-band, band pass and versatile Hybrid Filter Bank A/D, conversion for software radio", in IEEE TCAS-I (Transactions Circuits and Systems I), vol. 56, No. 8, August 2009.

[3] D. Asemani, J. Oksman and P. Duhamel "Subband architecture for Hybrid Filter Bank A/D converters", in IEEE Journal on selected Topics in Signal Processing, Vol.2, No. 2, pp.191-201, April 2008.

[4] Z. Song, C. Lelandais-Perrault, P. Benabes, "Synthesis of complex subband hybrid filter banks A/D converters using adaptive filters", in The 16th IEEE International Conference on Electronics, Circuits, and Systems, December 2009, accepted.

[5] S. Haykin(Editor), "Adaptive Filter Theory" Prentice Hall, 2002.

[6] D. Poulton, "Anti-aliasing Filter in Hybrid Filter Banks", in IEEE Second International Symposium on Communications, Control and Signal Processing, March 2006.

[7] P. Löwenborg, and H. Johansson, "Quantization noise in filter bank analog-to-digital converters", in IEEE International Symposium on Circuits and Systems, May 2001.

[8] Z. Zhang, G. Schmer "Analysis of Filter Coefficient Precision on LMS Algorithm-Performance for G.165/G.168 Echo Cancellation", in Application Report, Texas Instruments, February 2000.

[9] C. Lelandais-Perrault, Z. Song, D. Poulton, P. Benabes, "Hybrid Filter Bank A/D conversion systems applied to future telecommunication scenarios", Joint IEEE-NEWCAS and TAISA Conference, June 2009. 